Musées, Patrimoine et Culture scientifiques et techniques

$178 \mid 2018$

juillet-août 2018

\title{
La tête dans les étoiles, un projet culturel mené en prison
}

Stéphanie Vergnaud

\section{OpenEdition \\ Journals}

Édition électronique

URL : http://journals.openedition.org/ocim/2629

DOI : $10.4000 /$ ocim. 2629

ISSN : 2108-646X

Éditeur

OCIM

Édition imprimée

Date de publication : 1 juillet 2018

Pagination : 5-11

ISSN : 0994-1908

Référence électronique

Stéphanie Vergnaud, "La tête dans les étoiles, un projet culturel mené en prison », La Lettre de l'OCIM [En ligne], 178 | 2018, mis en ligne le 01 juillet 2019, consulté le 07 septembre 2019. URL : http:// journals.openedition.org/ocim/2629; DOI : 10.4000/ocim.2629

Ce document a été généré automatiquement le 7 septembre 2019.

Tous droits réservés 


\title{
La tête dans les étoiles, un projet culturel mené en prison
}

\author{
Stéphanie Vergnaud
}

La conférence de Daniel Kunth sur l'histoire de l'astronomie a constitué le premier volet de l'expérience menée au centre pénitentiaire de Liancourt.

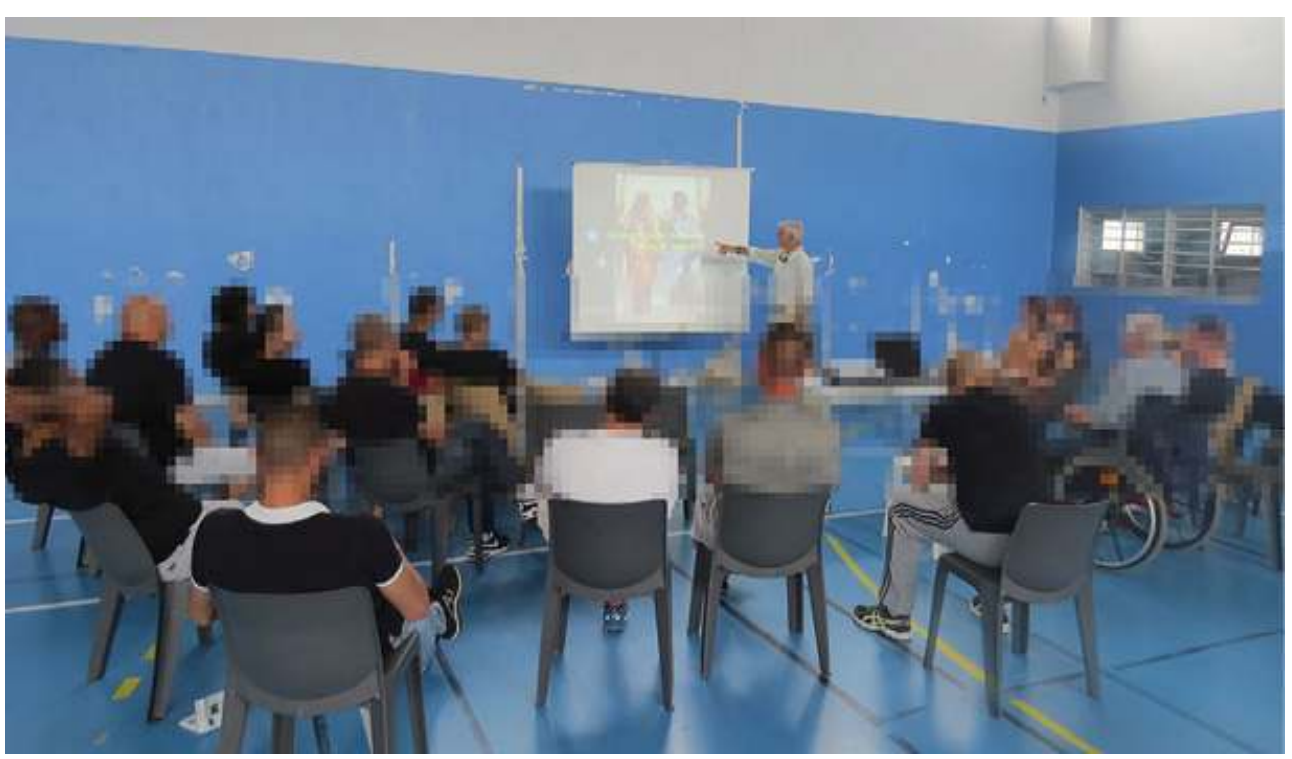

(c) Arnaud Debeve

1 "L' atelier m'a permis de m'évader", ce témoignage est fréquent de la part des détenus à la fin d'un projet artistique. Ces mots, ordinaires dans le cadre d'un processus de création, prennent une résonance toute particulière en prison. Cette phrase incarne le point principal de crispation entre les représentations sociales de la prison et les objectifs des institutions culturelles. En effet, les intentions de ces dernières - à savoir l'ouverture d'esprit, l'apport de connaissance, l'estime de soi, la valorisation de l'imaginaire paraissent encore subversives dans ce lieu d'enfermement et de tensions. Pourtant certaines missions de l'administration pénitentiaire et des institutions culturelles se 
rejoignent car, dans les deux cas, l'enjeu est de tisser du lien entre le dedans et le dehors, entre le soi et l'autre.

2 Cet article rend compte des résultats du projet de médiation culturelle mené par le Louvre-Lens au Centre pénitentiaire de Liancourt. Intitulé La tête dans les étoiles, il s'est déroulé du 19 juin au 7 juillet 2017, et associait des conférenciers scientifiques, des intervenants culturels et quatorze détenus entre 25 et 60 ans autour du thème de l'astronomie. Outre des conférences, dont une séance de planétarium, il s'agissait également de créer une œuvre collective, destinée à être exposée dans la nef principale du centre de détention. Ce projet s'inscrit dans la lignée des protocoles d'accord signés conjointement par les ministères de la Culture et de la Justice, le Louvre-Lens et la Direction Interrégionale des Services Pénitentiaires du Nord-Pas de Calais, Picardie et de la Haute Normandie.

3 Au-delà d'une revue des pratiques, nous chercherons à définir en quoi une telle action culturelle impulse de nouvelles pratiques carcérales et de nouvelles représentations de la détention. Un tel projet peut-il apporter une nouvelle conception des rapports sociaux ? La participation à ce type d'activité transforme-t-elle le regard que le détenu porte sur la culture? Les ateliers artistiques permettent-ils une reconstruction de soi ?

4 Pour répondre à ces interrogations, cet article décrit, dans un premier temps, le processus d'élaboration du projet, dans un deuxième temps, analyse le déroulement des différentes activités. Les résultats présentés proviennent des observations lors des différentes séances, des témoignages recueillis auprès des médias et des questionnaires administrés aux participants : quatorze questionnaires distribués, huit complétés lors de la dernière séance d'atelier, un envoyé par mél.

\section{Élaboration d'un projet en détention}

5 Depuis quelques années, des musées des Hauts-de-France interviennent dans les établissements pénitentiaires. Avant même l'ouverture du Louvre-Lens, la Maison du projet du Louvre-Lens et l'association Hors cadre avaient mis en œuvre un projet culturel interdisciplinaire avec les centres pénitentiaires de la région Nord-Pas de Calais: développé en 2012, dans les prisons de Bapaume, Longuenesse, Maubeuge et Valenciennes, Le Temps en Chantier avait pour but de sensibiliser des personnes détenues à l'ouverture prochaine du Louvre-Lens ${ }^{1}$. À partir de 2014, le service des publics du LouvreLens a développé plusieurs partenariats avec l'administration pénitentiaire.

\section{Définir une thématique transversale}

6 Malgré les initiatives, il s'avère que l'intervention en prison nécessite encore et souvent d'être argumentée. Pourquoi un musée se déplace-t-il en prison? Les formes de justification s'appuient sur une vision de la culture comme une liberté individuelle, un moyen de réconciliation avec soi, ou enfin un moyen d'intégration au collectif. Autrement dit, "la culture est un droit pour tous les citoyens, un moyen de développement personnel et un outil (re)tissant du lien, (re)construisant des identités, valorisant les personnes là où elles sont stigmatisées" (art. 27 de la Déclaration des Droits de l'Homme). Par conséquent, les objectifs d'un tel projet sont à la fois de donner accès à la culture à un public empêché, d'apporter des connaissances, de faire entrer l'extérieur à l'intérieur, d'éveiller l'esprit 
critique, et de (re)valoriser l'estime de soi. Mais si la réponse au pourquoi semble évidente pour un service de médiation, il faut surtout répondre à la question comment? Comment élaborer un projet collectif, sans connaître au préalable les profils des participants? Pouvons-nous établir un profil type ? Bien évidemment, cela est impossible, voire malheureux. Comment alors se rapprocher de la réalité des uns et des autres? Comment tisser du lien entre les collections classiques d'un musée d'art et les préoccupations quotidiennes des détenus? Le postulat de départ considérait donc des profils socio-culturels diversifiés avec comme points communs deux notions : celle de la rupture et celle de l'enfermement.

7 Plusieurs objets des collections semi-permanentes exposées au Louvre-Lens offrent une perspective temporelle et géographique large sur le thème de l'observation du ciel $^{2}$. Ce thème nous a semblé pertinent à explorer dans le contexte d'un centre de détention. Il permettait de discuter sur les échanges scientifiques et mythologiques entre l'Orient et l'Occident et d'évoquer le rapport entre science et religion - deux sujets qui constituent indéniablement des crispations au sein d'une prison. De plus, entre des murs, "le ciel n'estil pas l'un des rares espaces de projections au sein duquel on peut encore se raconter des histoires

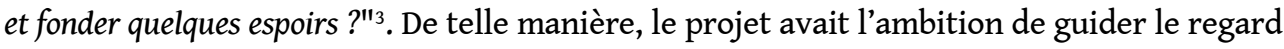
des détenus vers un ciel interrogé depuis l'Antiquité, par les sciences, les religions et l'art à travers le monde. Par sa transversalité, il visait également à libérer la parole des détenus et à leur faire expérimenter la capacité de faire aboutir collectivement un projet de création.

Des objets patrimoniaux (boîte aux planètes, coupe magique, globe céleste) présentés à la galerie du temps au Louvre-Lens ont été une source d'inspiration pour le second volet de l'expérience encadré par la plasticienne Justine Van Den Driessche.

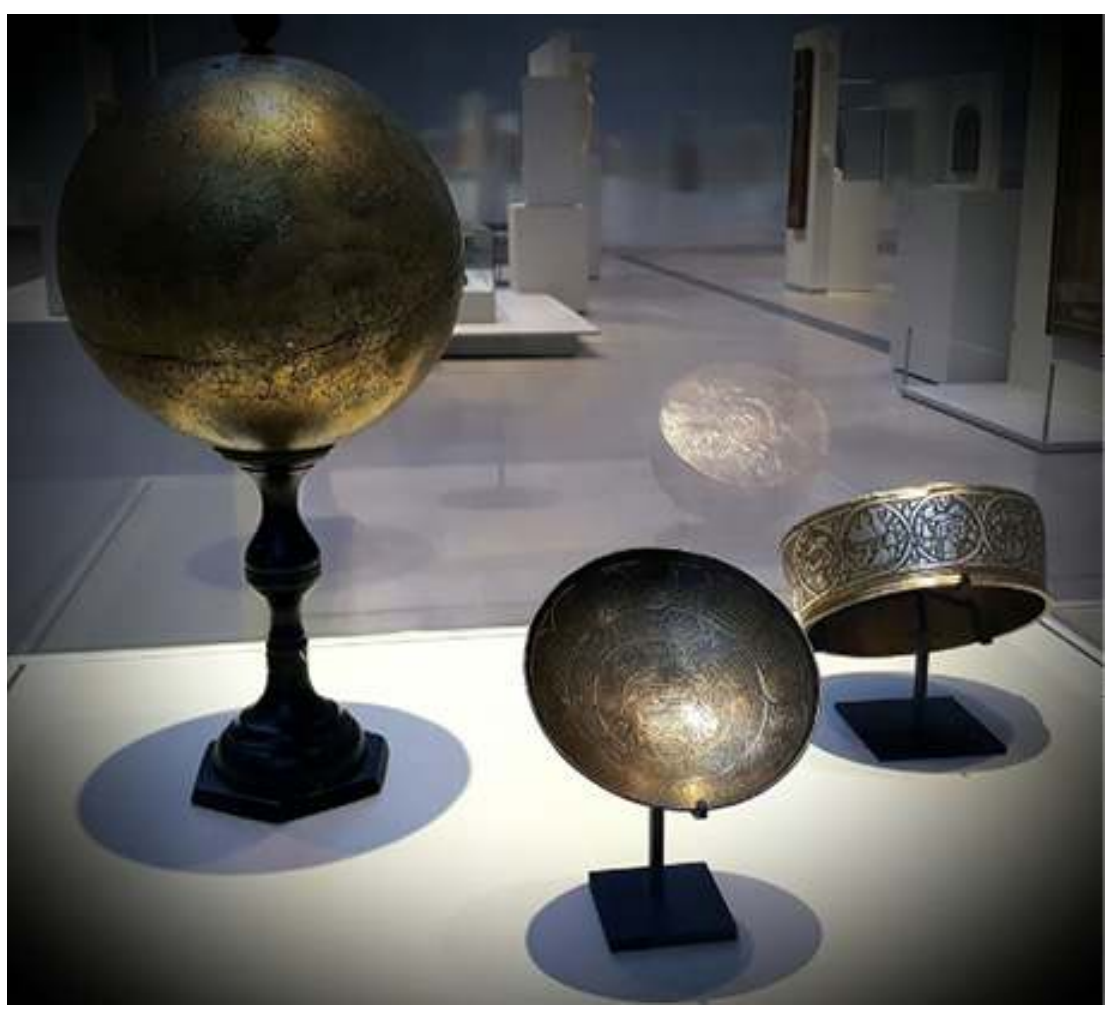

(c) Stéphanie Vergnaud 


\section{Anticiper les réticences par la collaboration}

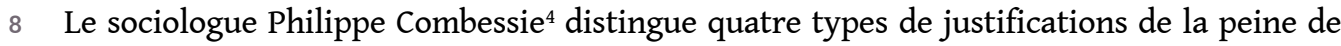
prison. Selon les trois premiers, le plaisir inhérent à un projet artistique n'a pas lieu d'être dans ce lieu d'enfermement. En effet pour certains, la prison est le lieu de la privation et de l'expiation censée compenser la souffrance que le détenu a générée. Pour d'autres, l'objectif de dissuasion de la récidive implique la peur des sanctions. Enfin pour d'autres, la prison ne permet que la neutralisation temporaire du coupable. Selon ces trois arguments, soit l'apport de culture ne changera rien, soit les détenus ne sont pas légitimes à recevoir ce moment d'évasion. Le développement d'activités culturelles dans les prisons requiert donc la quatrième justification de la peine, celle d'un temps possible de réadaptation à la société. En ce sens, les conseillers pénitentiaires d'insertion et probation (CPIP) et les coordinateurs de projet cherchent à rompre l'hermétisme des prisons et à changer le regard posé sur les détenus.

9 La mise en œuvre de ce projet a été grandement facilitée grâce à l'implication de l'administration pénitentiaire dès son élaboration. Les premiers contacts entre Le LouvreLens et la direction de la prison de Liancourt ont été pris à l'occasion de la signature de la convention entre le musée et la DISP le 16 mars 2016. La première réunion dans les locaux de la prison, en octobre 2016, a permis à l'équipe du musée de découvrir les différents espaces de l'établissement et également rencontrer certains personnels pénitentiaires : le centre pénitentiaire de Liancourt a une capacité de 614 places, il comprend deux quartiers de détention hommes majeurs, une maison d'arrêt hommes, un quartier de détention mineurs, un quartier d'accueil des arrivants et un quartier de vie.

L'ambition du projet autour de l'astronomie a vite révélé la nécessité de solides connaissances dans différents domaines scientifiques. Afin de s'assurer de la légitimité d'une telle proposition thématique, le recours à des professionnels issus de différentes disciplines était impératif. L'interdisciplinarité était considérée comme un atout. Implanté dans la métropole lilloise depuis vingt ans, le Forum départemental des sciences de Villeneuve d'Ascq a été mobilisé afin d'apporter la caution scientifique nécessaire à la réussite du projet. Le médiateur scientifique du Forum Département des Sciences, André Amossé, a notamment proposé de faire une présentation du ciel dans un planétarium nomade. Pour renforcer cette caution scientifique, un astrophysicien de l'Institut d'Astrophysique de Paris, Daniel Kunth, était sollicité pour une conférence sur l'histoire de l'astronomie. Pour le second volet de ce projet, à savoir la réalisation d'une œuvre collective, une proposition a été formulée par la plasticienne Justine Van den Driessche. Les motivations des artistes et des scientifiques peuvent s'expliquer par le fait que le travail en prison permet de partager leur connaissance, de renouveler leur pratique artistique, de faire naître de nouveaux référents et formes d'expression.

11 Enfin, au début de l'année 2017, une rencontre était organisée avec les mécènes de la Fondation AnBer ${ }^{5}$, afin de leur soumettre le projet qui a reçu un accueil favorable. Le projet a été soutenu dans sa totalité.

\section{Au cœur de l'échange et du processus créatif}

12 Entrer dans les murs d'une prison, c'est parcourir des perspectives visuelles et sonores arrêtées. Mener un projet culturel en prison c'est composer avec de nombreuses 
contraintes et incertitudes relatives à la sécurité, l'appropriation du projet par les détenus et enfin sa réception et son accompagnement par le personnel pénitentiaire.

\section{L'observation du ciel}

13 Le cycle a commencé le 19 juin 2017. Aucun espace d'une prison n'est véritablement destiné à accueillir des activités artistiques ou des conférences. Dans la matinée, André Amossé a donc installé le planétarium nomade dans le gymnase du centre pénitentiaire. La température dépassait les 30 degrés à l'extérieur. Par conséquent, les conditions d'observation à l'intérieur de la structure gonflable, s'annonçaient étouffantes. Nous avons également installé le dispositif pour les conférences dans le gymnase. Avec l'aide de quelques gardiens et détenus, une vingtaine de chaises en plastique ont été disposées devant un écran, placé à l'entrée de cette immense salle. L'abondance de lumière perturbait un peu la visibilité des images projetées. Néanmoins encadrants, intervenants et détenus étaient avisés de ces compromis.

Le planétarium itinérant du Forum départemental des sciences de Villeneuve d'Ascq a permis aux détenus de découvrir la voûte céleste.

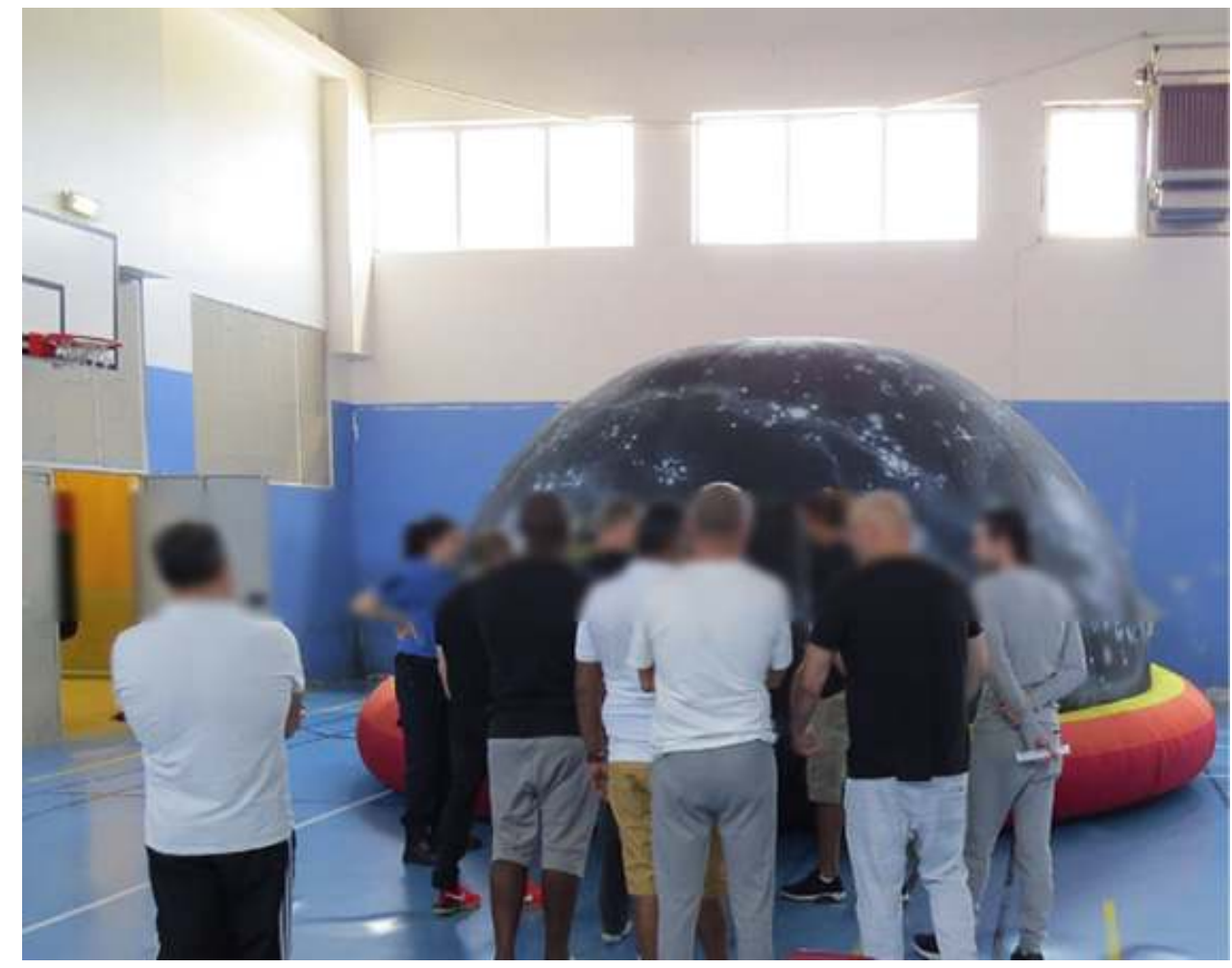

(c) Stéphanie Vergnaud

14 À 14 heures, la séance a commencé. Après une présentation générale du projet et des intervenants, la séance de planétarium inaugurait le cycle. Nous avons estimé que l'impact immédiat d'un tel dispositif nous permettrait d'établir rapidement un climat de confiance. André Amossé a commencé par donner quelques consignes au sujet du planétarium. L'un des participants en fauteuil roulant devait notamment entrer en premier en passant sous la structure. Les autres, impatients, se sont laissés aspirer, avec enthousiasme, mais sans précipitation, dans la structure. Assis ou allongés le long des 
parois, les yeux tournés vers la voûte du planétarium, chacun a trouvé rapidement sa place. La lumière baissait au rythme accéléré d'une journée. Dans le noir et dans le calme, André Amossé pouvait commencer sa présentation du mouvement des étoiles, de la lune et du soleil. Certains participants n'ont pas hésité à poser des questions pendant la présentation, sur le nom des étoiles, la taille des planètes ou la vie extraterrestre. Un échange entre détenus confirmait la complexité et la sensibilité de la thématique. L'un deux demandait : "la bonne distance de la terre au soleil est-elle due au hasard ou existe-il une sorte de grand horloger ?". Un autre détenu lui a répondu : "là, il te présente un propos scientifique". Une question qui a eu le mérite d'ouvrir le débat, et répondait à notre attente d'éveiller l'esprit critique. La séance s'est poursuivie avec la conférence du Louvre-Lens par la médiatrice culturelle, Stéphanie Vergnaud. Après la présentation des œuvres sélectionnées, elle a présenté la Galerie du Temps où elles sont disposées à travers un film de communication. Les réactions et questions ont été multiples, certains détenus s'interrogeaient sur l'ancienneté de l'observation du ciel, d'autres s'intéressaient à l'histoire mythologique des constellations, et certains partageaient avec le groupe ce qu'ils avaient lu ou vu dans des émissions scientifiques.

Lors des au revoir les réactions étaient unanimement positives : "vous avez réussi à nous libérer l'esprit ; pari gagné pour nous mettre la tête dans les étoiles; c'est un sujet qui parle à tout le monde".Le lendemain, le 20 juin 2017 a eu lieu la conférence de l'astrophysicien Daniel Kunth, L'origine des constellations et les mots $d u$ ciel. Lors des premières minutes, le conférencier a eu quelques difficultés à capter l'attention des participants, quelques apartés se faisaient entendre. Lui-même semblait avoir quelques difficultés à gérer la position de son corps dans cette configuration inhabituelle et la chaleur toujours étouffante. Toutefois, il parvint à détendre l'ambiance avec son explication amusée du terme de canicule, terme emprunté aux constellations. À la fin de la conférence, Daniel Kunth a répondu à plusieurs questions comme la distance des étoiles, l'existence d'une planète faite de diamant, le temps nécessaire pour aller sur Mars... Des échanges qui se sont poursuivis pendant le rangement du matériel. 
Mise en place de l'atelier artistique sous la conduite de Justine Van Den Driessche.

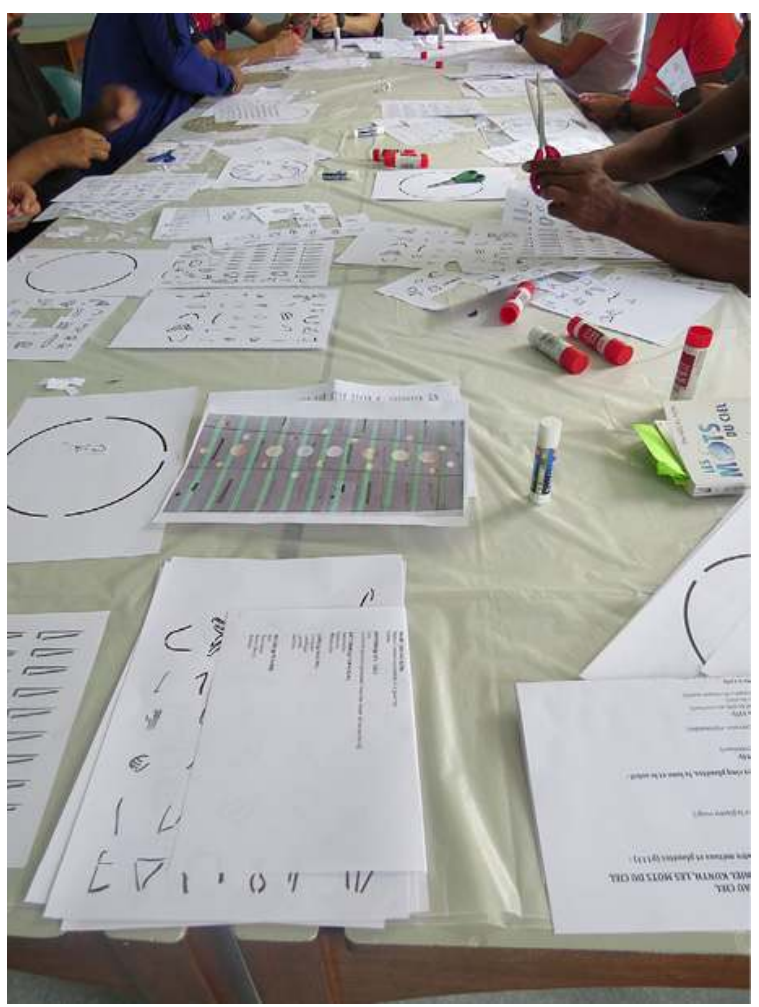

(c) Stéphanie Vergnaud

\section{L'atelier artistique}

Le projet s'est poursuivi à travers huit séances d'atelier, menées par l'artiste Justine Van Den Driessche, du 21 juin au 7 juillet 2017.

La réalisation d'un atelier doit composer avec quelques contraintes et réticences. La plus évidente est celle des outils et matériaux autorisés à entrer en détention. Les surveillants sont souvent très méfiants lors des interventions artistiques, craignant le vol d'outils, l'échange d'objets ou les mouvements de personnes dans les couloirs. Autant de risques pour la sécurité. Néanmoins, ce projet a bénéficié de l'engagement de l'administration pénitentiaire qui a favorisé l'entrée, sans difficulté, d'outils jugés dangereux (ordinateurs, ciseaux, colles) et la bonne circulation des intervenants. Les ateliers se sont déroulés dans une salle exiguë, offrant une perspective directe sur la cour de promenade, avec laquelle elle partageait la chaleur et les bruits. Cependant, la salle avait l'avantage de posséder six tables réunies au centre, un petit point d'eau et une commode à laquelle un gardien a ajouté un cadenas.

Une autre difficulté, moins visible, mais tout aussi importante à prendre en compte, est l'indifférence possible ou la méfiance des surveillants pénitentiaires. Beaucoup ne comprennent pas l'intérêt que ce type d'activité peut susciter, ne perçoivent pas les possibilités d'insertion qu'elles pourraient créer. Elles peuvent être perçues comme une injustice ou une absurdité. Il faut souligner que les surveillants n'ont pas toujours pleinement accès à l'art eux-mêmes et qu'une "défiance réciproque" existe entre détenus et gardiens ${ }^{6}$. Ces difficultés se sont traduites par quelques retards de plusieurs détenus 
aux activités, certains surveillants tardant à ouvrir les portes des cellules, ou à conduire les détenus aux salles d'atelier ou parfois oubliant l'un d'eux... D'autres profitent de l'occasion pour asseoir leur autorité par la menace. Aucun incident n'est toléré, sous peine de voir les ateliers interdits. C'est par cet avertissement que la première séance d'atelier a débuté.

Croquis préparatoire réalisé sous la forme de collage.

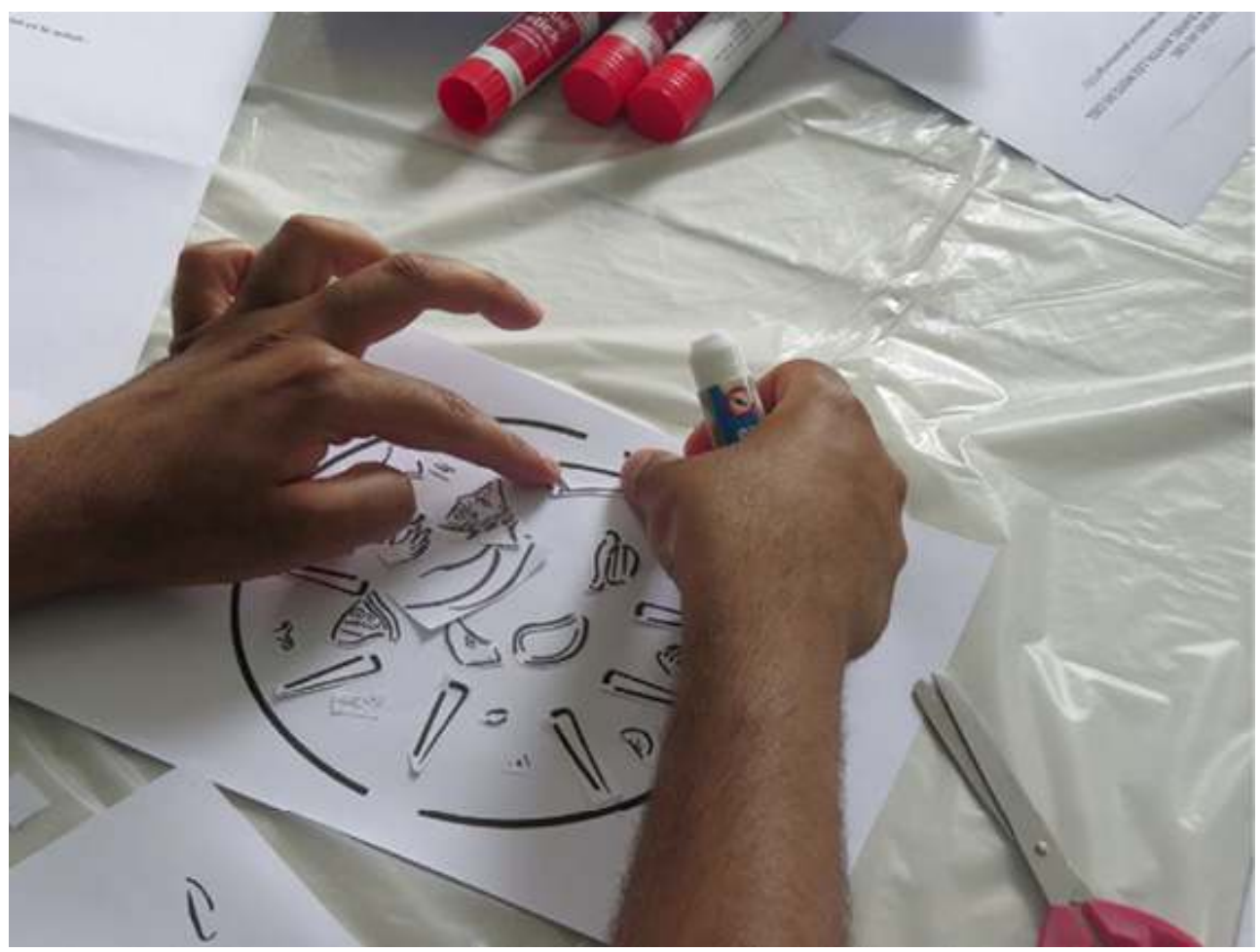

(c) Stéphanie Vergnaud 


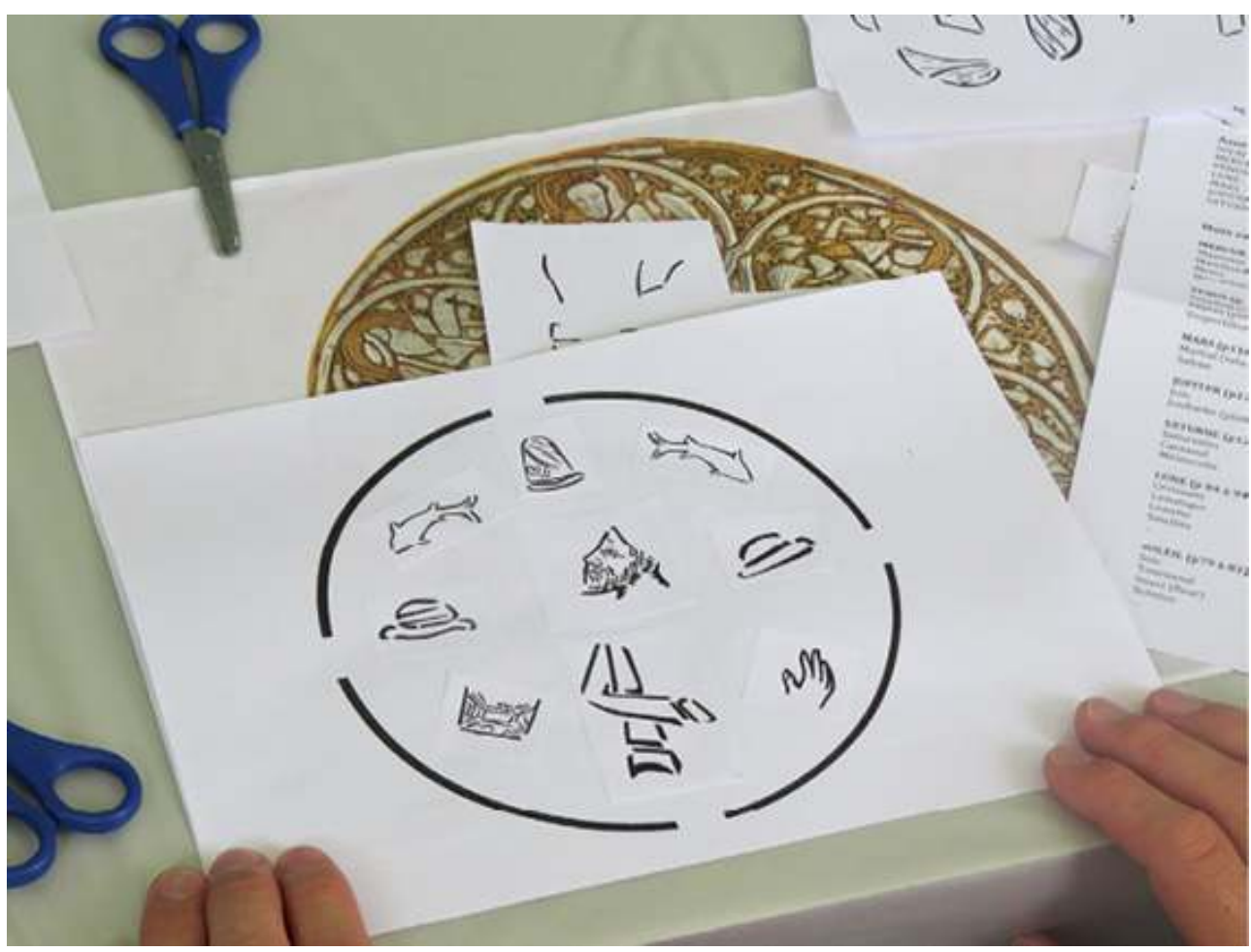

(C) Stéphanie Vergnaud

Toutefois comme l'indique la psychologue Caroline Legendre, "la création, nécessité existentielle, fondamentale et structurale pour l'être humain, le serait de façon d'autant plus cruciale et vitale dans l'espace-temps carcéral marqué par la rupture, la séparation, le manque, la perte, l'absence"7. En effet, la création permet de construire l'autonomie et favorise l'expression personnelle, difficile entre les murs déresponsabilisants de la prison. L'indifférence, le contrôle du courrier, les visites surveillées, l'isolement, pèsent sur le détenu qui retrouve dans la création un désir d'exister pour soi et envers les autres. L'expression artistique permet de formuler un message, une idée, une émotion, en toute liberté.

Justine Van Den Driessche a donc cherché naturellement à réduire les difficultés et à encadrer la création, sans déposséder les détenus de leur liberté de création. À l'aide des techniques du collage, de la peinture et du pochoir, les participants ont reproduit sur sept grands panneaux de bois circulaires (61 centimètres de diamètre) les personnages représentés sur la boîte aux planètes du Louvre. Ils ont également inventé leur propre figure sur quatorze panneaux de 31 centimètres de diamètre, à partir de la fragmentation des formes qui composaient les sept figures de l'objet étudié et des mots extraits du livre de Daniel Kunth. Il ne s'agissait pas d'avoir un regard condescendant et de priver les détenus d'un regard d'appréciation. Au contraire, l'exigence de qualité était primordiale pour atteindre les objectifs du projet. "À travers l'art, c'est un moyen d'évasion pour pouvoir s'échapper du quotidien et puis d'aller de l'infiniment petit à l'infiniment grand, entre la minutie de la touche de peinture qui nous permet de nous projeter jusqu'au ciel et aux étoiles", explique l'artiste ${ }^{8}$. Ainsi, les participants se sont lancés sans hésitation dans la découpe des formes et chacun s'est approprié très facilement le sujet, acceptant d'expliquer sa composition devant le groupe : 
"J'ai mis deux têtes pour montrer les deux natures de l'homme, l'une de face, l'autre de profil... avec un bouclier de protection... qui s'y frotte si pique".

"Je l'ai appelé Joie... il y a dieu et la porte du ciel, les miracles avec le pain et les poissons".

"Je l'ai appelé Lunatique car je l'ai fait au feeling".

"Le titre est Souci pour les soucis du quotidien, c'est une galère et les fleurs qui s'appellent soucis. Ce mot est positif ou négatif".

"Ça s'appelle Divinité en référence au Dieu, à la vie et à la lumière, je ne voulais pas écrire le mot Dieu car ici c'est compliqué".

"J'ai écrit Big Bang juste là, et en gros j'ai mis des petits trucs un peu partout pour montrer une explosion et ça va un peu dans tous les sens. Ça représente bien une création"9.

"Je n'ai pas de pression. Si on dit que mon dessin est moche, je dirai que ce n'est pas le mien"10.

Le partage d'un intérêt et d'un objectif communs, la nécessité de se côtoyer, de travailler collectivement a impliqué que les participants échangent, voire parfois s'entraident, une dynamique collective rare en prison. Le sentiment de s'instruire a laissé place à celui de se divertir dans un moment considéré comme privilégié. Les rires étaient nombreux.

Le 7 juillet 2017 une restitution du projet a été organisée dans la salle de culte. Un moment essentiel pour marquer l'investissement et l'accomplissement d'un travail, notions importantes dans le processus de réinsertion. "Dans une salle (sous surveillance!) des hommes qui semblent comme vous, comme moi, accueillants, sympathiques, parfois timides, aux regards clairs, heureux d'avoir pu "S'ÉVADER", le temps d'un atelier artistique, de leur vie d'enfermement, de leur vie de coupables. D'avoir droit à d'autres regards sur eux. Fiers aussi d'avoir réussi à créer une œuvre collective qui parle des étoiles, du lien Orient/Occident... envie de venir découvrir les œuvres (pour de vrai) quand ils sortiront... dans 5 ans et si je suis "sage" dans 4 ans $1 / 2$ !", a témoigné Karine Desombre ${ }^{11}$. En effet, face aux félicitations et aux remerciements, un sentiment de fierté a émergé dans la contemplation de l'œuvre réalisée, un regard positif sur soi et sur le groupe. La reconnaissance d'autrui, des intervenants, mais aussi de la direction du musée, du centre pénitentiaire et des mécènes offre aux détenus un moment privilégié. L'avis des professionnels, considéré comme exigeant, apparaît aux yeux des détenus, plus aptes à porter un jugement objectif, à évaluer des compétences. "Monsieur, gardez mon tableau pour votre collection. Dans cinq ans, il vaudra des millions sur le marché de l'art. Il est beau, non ?" fanfaronne James ${ }^{12}$. Il l'offrira au conservateur du musée, Luc Piralla. La médiatisation du projet et ce moment de restitution ont donné une visibilité aux participants et favorisé le sentiment d'accomplissement de soi. 
Mise en couleurs du panneau.

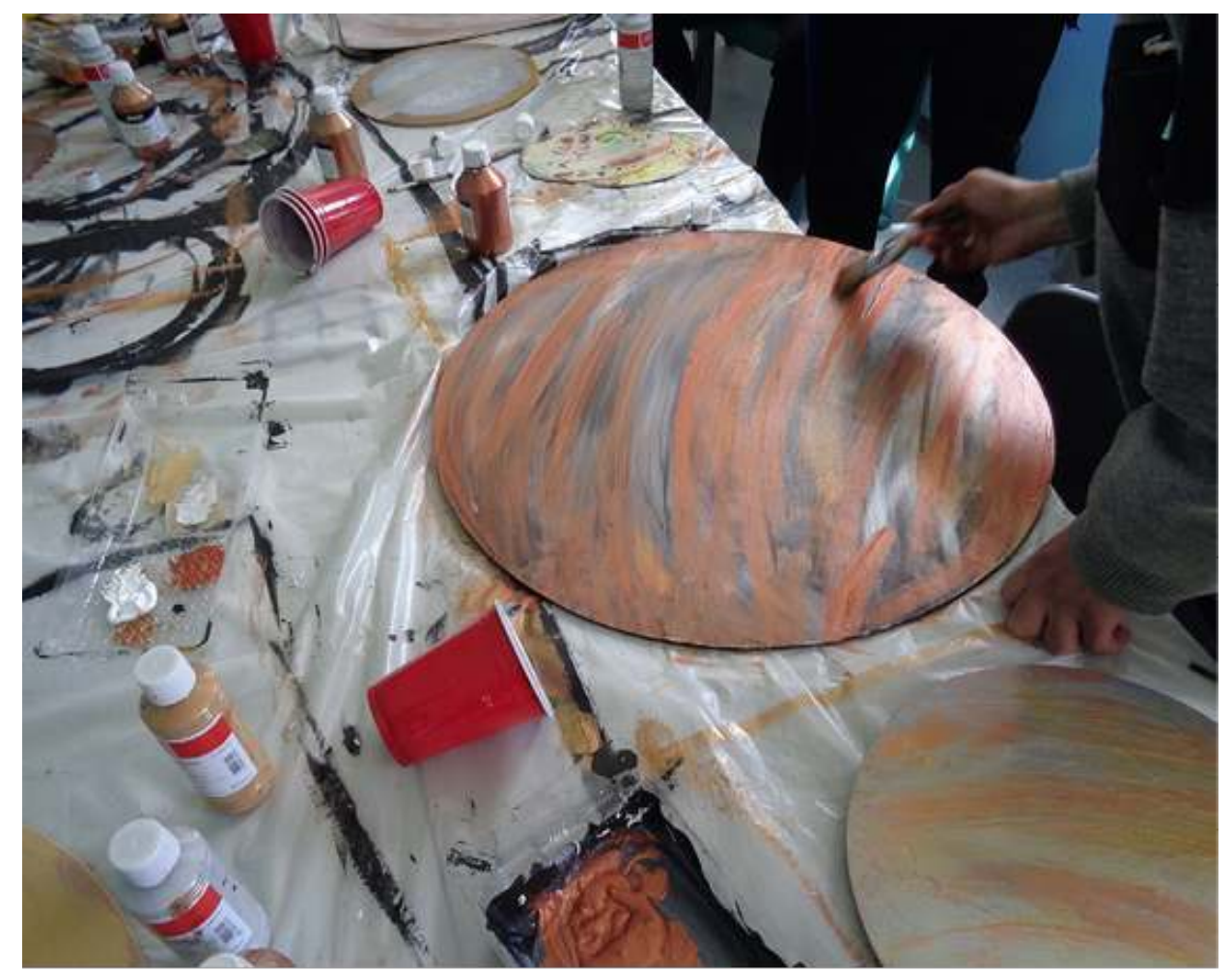

(c) Justine Van Den Driessche

\section{Bilan}

Au final, le projet a réuni quatorze hommes, un taux de participation rare en prison. Il faut noter qu'il existe un certain nombre de règles limitant l'accès des détenus aux activités artistiques : les personnes, maintenues au quartier d'isolement ou DPS (détenus particulièrement signalés), le travail, la formation professionnelle, le sport, les horaires de parloirs, d'avocat ou les rendez-vous médicaux. En outre, les motivations de participation sont diverses et multiples. Dans notre cas, l'enquête révèle qu'elles sont liées tout autant à l'activité proposée (la thématique de l'astronomie et le travail collectif), qu'à l'opportunité de discuter avec des intervenants extérieurs. Certains admettent qu'il s'agit simplement de satisfaire des besoins sociaux, comme s'échapper quelques heures de sa cellule, et occuper le temps : "On s'inscrit à tous les ateliers. On est un petit groupe motivé. Que des gens viennent de dehors pour nous, cela fait plaisir. Et puis cela fait sortir de la cellule", reconnaît Elvis. "Avec les barreaux et le grillage, cela fait des années que je n'ai pas vu les étoiles", soupire un autre participant. Petit, il rêvait d'être astronaute. "Les choses ont mal tourné", résume-t-il pudiquement ${ }^{13}$. "Ça montre qu'on peut faire travailler notre imagination, ça nous sort du quotidien et surtout on s'entretient avec des gens de l'extérieur. Ça nous permet vraiment de penser à autre chose et d'avoir un lien avec l'extérieur, surtout artistique ", témoigne Shim devant la caméra de France $3^{14}$. En outre, l'engagement volontaire et sur le long terme des détenus dans un projet culturel est variable. Ici, le groupe est resté relativement stable jusqu'à la fin, seul un participant a abandonné l'atelier. 
31 L'action culturelle en prison peut participer à créer des espaces dénués de tous enjeux affectifs, de toutes questions liées à l'affaire pénale des détenus. Les témoignages précédents révèlent que ces espaces de liberté permettent de rompre avec la rigueur et la circularité du temps carcéral, un temps libéré des contraintes, un temps interpersonnel. En cela, chaque intervenant s'est efforcé de ne pas réduire les participants à leur statut de détenu. Ils se sont appuyés sur leur professionnalisme et leur pédagogie afin d'instaurer des relations égalitaires avec les participants. De part leur simplicité et leur convivialité, les intervenants sont parvenus à faire circuler la parole. Les détenus ont établi des échanges authentiques avec les intervenants, n'hésitant pas à poser des questions et à partager leurs connaissances. L'analyse des questionnaires indique que le sentiment qui a prédominé chez les participants suite aux conférences est celui d'avoir appris quelque chose : à la question "quelles sont les impressions ressenties lors de la séance ?" la totalité des participants ont répondu "Instruit / curieux" ; à la question "quel est l'aspect qui a retenu le plus votre attention?" la totalité ont répondu "les explications scientifiques sur les étoiles et les planètes" et la majorité ont répondu "l'aspect historique et sociologique des œuvres". Les détenus ont notamment attaché une grande importance aux explications scientifiques et historiques. En tant qu'acteurs créateurs, ils ont accepté de participer sans toujours savoir où ils allaient, se sont adaptés, ont échangé et ont formé un collectif capable de produire une œuvre commune.

Toutefois, l'enquête révèle plusieurs amélioration possibles. La vidéo du musée a suscité l'intérêt des détenus, mais elle a été jugée trop courte. Ils ont également apprécié la mise à disposition des ouvrages de Daniel Kunth et du musée, mais ont formulé le souhait d'avoir plus de supports à conserver en cellule. Il faut noter qu'il existe une bibliothèque par quartier, les ouvrages circulent, sans doute difficilement, entre les bibliothèques. Par ailleurs, pour favoriser l'immersion pour le public éloigné de la culture, le Louvre-Lens teste la mise en place d'une visite virtuelle dans laquelle à l'aide d'une manette et face à un écran, les participants peuvent diriger le robot, visiter les espaces du musée, et interagir en direct avec un médiateur et les visiteurs.

De ces constats, retenons qu'une telle action nécessite de privilégier une approche immersive, tant par la multiplication des supports de médiation que par l'œuvre collective. Le deuxième axe d'amélioration est la temporalité de l'enquête. L'évaluation de l'impact d'un tel projet sur la reconstruction de l'individu implique de pouvoir comparer les résultats. Cela suppose d'une part le suivi d'un échantillon d'individus sur le long terme. D'autre part, cela nécessite l'élaboration de critères d'évaluation partagés par les différents intervenants extérieurs et les CPIP. L'action a provoqué des dynamiques relationnelles fortes entre les intervenants et les participants et des collaborations nouvelles entre institutions. Le projet a bousculé à la fois les représentations sociales que les intervenants extérieurs peuvent avoir de ce monde clos, ainsi que celles des détenus envers les institutions officielles. Ainsi, cette action a participé à un redimensionnement du champ des possibles, tout en clarifiant la méthode à suivre. 
Quelques-uns des panneaux finis avec les personnages figurant sur la boîte aux planètes du Louvre.

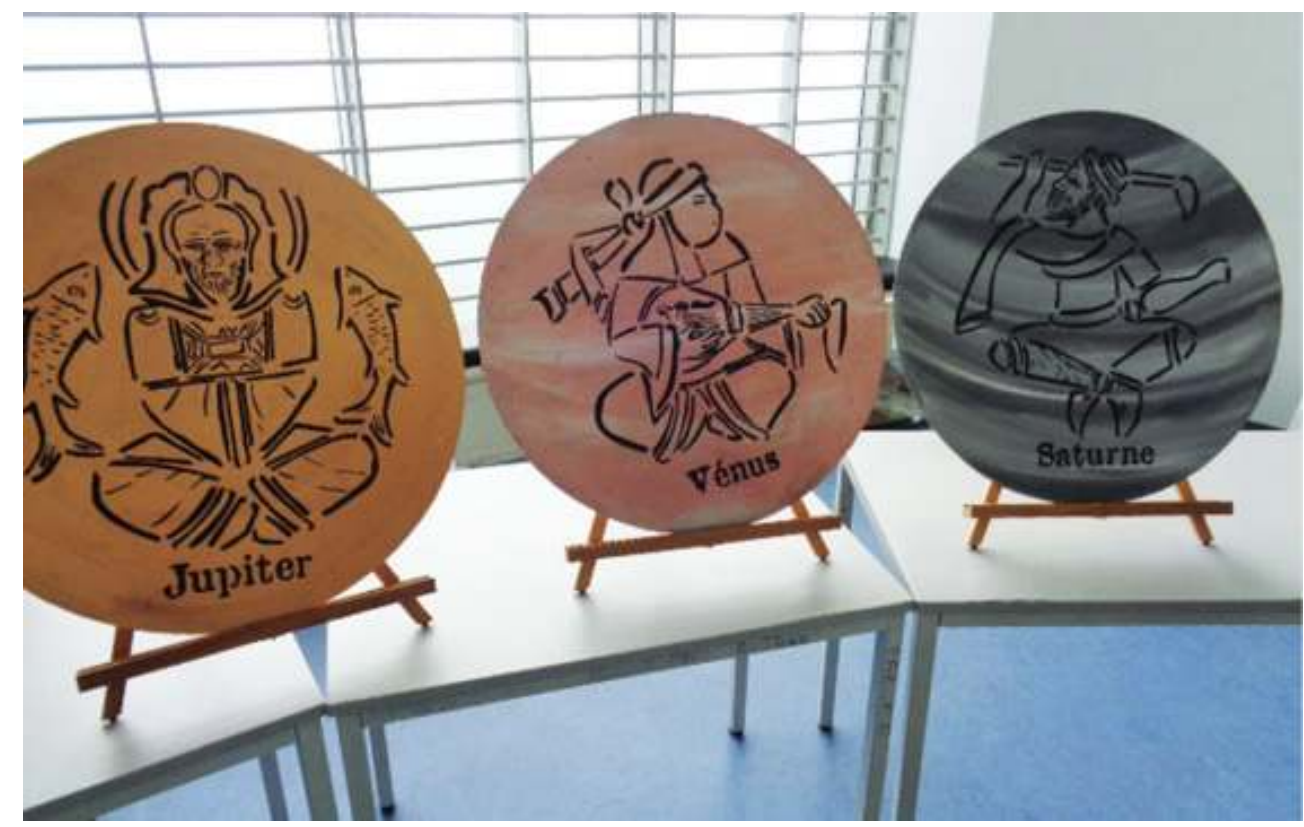

(c) Arnaud Debève

Combessie, P. La sociologie de la prison. La Découverte, 2009, 128 p.

Legendre, C. La création, une nécessité vitale, in Création et prison. Édition de l'atelier/

Éditions ouvrières, 1994.

Rostaing, C. La relation carcérale, identités et rapports sociaux dans les prisons de femmes. Paris : PUF, 1997.

Rostaing, C. et Touraut, C. Processus de création culturelle en prison : une innovation ordinaire ? Socio-logos, 2012 (en ligne) http://journals.openedition.org/socio-logos/2658. Siganos, F. L'action culturelle en prison. Pour une redéfinition du sens de la peine. Paris : L'Harmattan, 2008.

\section{NOTES}

1. http://horscadre.eu/

2. Les œuvres sélectionnées : Boîte aux planètes d'Égypte ou de Syrie (XIVe siècle) ; Coupe magique de Syrie représentant des constellations (XIIIe-XIVe siècles) ; Kudurru représentant les divinités astrales de Babylone (vers 1100 avant J.C.) ; Relief romain représentant le dieu du soleil iranien, Mithra (vers 100-200 après J.C.) ; Globe céleste de Maragha (vers 1315) ; Jardinière de Louis XVIII portant les signes du zodiaque (1819) https://www.louvrelens.fr/la-galerie-dutemps/les-oeuvres-de-la-galerie-du-temps

3. Dossier de présentation du projet de Justine Van den Driessche

4. Combessie, P. La sociologie de la prison. La Découverte, 2009, 128 p. 
5. Reconnue d'utilité publique, la Fondation AnBer, créée en 1989 par Bernadette et André Leclercq, soutient des actions de solidarité, en France et dans le monde entier, autour de thèmes liés à l'éducation, la santé et le travail.

6. Ce terme emprunté à la sociologue Corinne Rostaing s'explique par un rapport de force entre un personnel disposant d'un pouvoir légalement établi de contrôler et sanctionner et des détenus censés subir et obéir. Rostaing, C. La relation carcérale, Identités et rapports sociaux dans les prisons de femmes. Paris : PUF, 1997.

7. Legendre, C. La création, une nécessité vitale, in Creation et prison. Édition de l'Atelier / Éditions ouvrières, 1994, p. 23

8. France 3 Hauts-de-France, JT 12.13 Picardie, 6 juillet 2017.

9. Propos recueillis pendant les ateliers.

10. Heyligen, J. Liancourt : les détenus s'évadent en peinture avec le Louvre-Lens, Le Parisien, 06 juillet 2017.

11. Page Facebook de Karine Desombre, publication privée de juillet 2017

12. Propos recueillis pendant la restitution.

13. Heyligen, J. Liancourt : les détenus s'évadent en peinture avec le Louvre-Lens, Le Parisien, 06 juillet 2017.

14. France 3 Hauts-de-France, JT 12.13 Picardie, 6 juillet 2017.

\section{RÉSUMÉS}

Depuis plusieurs années, le Louvre-Lens s'est engagé dans une politique de médiation culturelle avec des centres pénitentiaires. Le projet autour de l'astronomie présenté ici suscite un certain nombre d'interrogations parmi lesquelles l'influence de cette action sur les pratiques carcérales, la nature du lien ainsi créé entre le dedans et le dehors et l'évolution du regard porté par les détenus sur le monde de la science et de l'art.

INDEX

Mots-clés : Société, inclusif, expérience

\section{AUTEUR}

\section{STÉPHANIE VERGNAUD}

Médiatrice culturelle au Louvre-Lens

stephanie.vergnaud@louvrelens.fr 